\title{
Special Issue on NLP \& Semantics
}

\author{
Daniel Hershcovich ${ }^{1} \cdot$ Lucia Donatelli $^{2}$
}

Accepted: 29 May 2021 / Published online: 2 October 2021

(c) Gesellschaft für Informatik e.V. and Springer-Verlag GmbH Germany, part of Springer Nature 2021

\section{Dear reader,}

Passing the Turing test, or exhibiting intelligent behavior indistinguishable from that of a human, is often cited as one of the major goals of Artificial Intelligence. However, demonstrating such behavior by means of interacting with natural language - the test's passing criterion-is sometimes considered too modest of a goal given current research. As powerful Natural Language Processing (NLP) technology continues to achieve human-like and often superhuman performance based on standard benchmarks, many questions regarding what we assumed our computers to be capable of are surfacing. Innovative techniques and models introduced at a staggering pace are shaking the scientific community in academia and industry alike.

As astonishment by our rapid progress grows, awareness of the limitations of current methods is entering the consciousness of more and more researchers and practitioners. A central difficulty much NLP research faces is how to generalise from controlled data sets to real-world environments that require a wider range of language and linguistic phenomena than data-specific and often superficial heuristics can account for. In addition to asking what our computers are capable of, NLP researchers are also asking questions about the fundamental relationship between language and intelligence and what makes either decidedly 'human'.

This special issue introduces diverse perspectives on current questions in NLP research. In the opening technical contribution of this special issue [4], we review the current state of semantics in NLP. We focus on the dichotomy between semantics, as formally defined in linguistics, and meaning, an extended understanding of semantics employed in much NLP research that integrates theoretical foundations of

Daniel Hershcovich

dh@di.ku.dk

Lucia Donatelli

donatelli@coli.uni-saarland.de

1 University of Copenhagen, Copenhagen, Denmark

2 Saarland University, Saarbrücken, Germany linguistic competence with usage-based performance effects resulting from contextual factors. This survey article sets the stage for the issue's articles, which approach the question of how to represent meaning from distinct perspectives.

In the article Ideal Words: a Vector-based Formalisation of Semantic Competence [2], Aurelie Herbelot and Ann Copestake bridge theories of semantic competence with semantic performance with a formal distributional account based on corpus data, arguing that the representation of meaning is in principle learnable from performance data and can be leveraged for teaching artificial agents meaning.

In their article Draw Mir a Sheep: A Supersense-based Analysis of German Case and Adposition Semantics [5], Jakob Prange and Nathan Schneider investigate the complex relationship between form and meaning, focusing on the German language, and specifically the little words (or parts of words) called prepositions and case markers, which nevertheless play a heavy role in sentence semantics.

In Embodied Human Computer Interaction [6], James Pustejovsky and Nikhil Krishnaswamy describe a simulation platform for building Embodied Human Computer Interaction (EHCI). The authors present a semantic formalism for multiomodal interaction and highlight the importance of incorporating modalities apart from language such as gesture, action, gaze, and facial expression into the common ground for enhanced interaction from both formal and computational perspectives.

In the paper Stance Detection Benchmark: How Robust Is Your Stance Detection? [7], Benjamin Schiller, Johannes Daxenberger and Iryna Gurevych analyze the robustness of models designed to detect the stance of a proposition toward a topic across diverse domains, an important task for fake news detection and argumentation.

The paper Designing a Uniform Meaning Representation for Natural Language Processing [9] by Gysel et al. presents a sentence-level and document-level symbolic meaning representation for many languages, developed in a large international project aiming at supporting lexical and logical inference at scale. 
In addition, Alexander Koller from Saarland University shared his expert opinions with us in an interview [3] on the utility of semantic representations, the fast-pace growth of the field of computational linguistics, and the challenges and joys of being a computational semanticist figuring out which hill to climb in NLU.

In their project report Do it Yourself but not Alone: Companion-Technology for Home Improvement-Bringing a Planning-Based Interactive DIY Assistant to Life [1], Pascal Bercher et al. describe an interactive assistant, ROBERT, that utilizes hierarchical planning and ontological reasoning to adapt to users and guide them through domestic projects.

Finally, the special issue includes abstracts of two recent $\mathrm{PhD}$ dissertations: in Learning High Precision Lexical Inferences [8], Vered Shwartz presents algorithms for recognizing semantic relations between words, and in The Syntax, Semantics, and Pragmatics of Japanese Addressee-Honorific Markers [10], Akitaka Yamada develops a discourse model in which the dimension regarding politeness-oriented expressions in Japanese is modeled as a Bayesian inference process.

Guest editors,

Daniel Hershcovich and Lucia Donatelli

\section{Content}

\subsection{Technical Contributions}

- It's the Meaning that Counts: The State of the Art in NLP and Semantics [4]—Daniel Hershcovich and Lucia Donatelli

- Ideal words: a vector-based formalisation of semantic competence [2]—Aurelie Herbelot and Ann Copestake

- Draw mir a sheep: A Supersense-based Analysis of German Case and Adposition Semantics [5]—Jakob Prange and Nathan Schneider

- Embodied Human Computer Interaction [6]—James Pustejovsky and Nikhil Krishnaswamy

- Stance Detection Benchmark: How Robust Is Your Stance Detection? [7]-Benjamin Schiller, Johannes Daxenberger and Iryna Gurevych

- Designing a Uniform Meaning Representation for Natural Language Processing [9]—Jens E. L. Van Gysel, Meagan Vigus, Jayeol Chun, Kenneth Lai, Sarah Moeller, Jiarui Yao, Tim O'Gorman, Andrew Cowell, William Croft, Chu-Ren Huang, Jan Hajic, James H. Martin, Stephan Oepen, Martha Palmer, James Pustejovsky, Rosa Vallejos and Nianwen Xue

\subsection{Interviews}

- Climbing the Hill of Computational Semantics [3]Interview with Alexander Koller, Saarland University

\subsection{Project Reports}

- Do it Yourself but not Alone: Companion-Technology for Home Improvement - Bringing a Planning-Based Interactive DIY Assistant to Life [1]—Pascal Bercher, Gregor Behnke, Matthias Kraus, Marvin Schiller, Dietrich Manstetten, Michael Dambier, Michael Dorna, Wolfgang Minker, Birte Glimm and Susanne Biundo

\subsection{Doctoral Dissertations}

- Learning High Precision Lexical Inferences [8]—Vered Shwartz

- The syntax, semantics, and pragmatics of Japanese addressee-honorific markers [10]—Akitaka Yamada

\section{Service}

\subsection{Journals and Conferences}

The field of NLP is inherently interdisciplinary: traditionally there is a distinction between Computational Linguistics, focusing on the investigation of linguistic research questions through corpora and computational methods; and NLP, focusing on developing technology to enable linguistic analysis, human-computer interaction and assistive tools. However, the borders are becoming more and more vague, as advanced technology is increasingly used in linguistics, and scientific investigation of the linguistic principles behind NLP technology is becoming more important and prevalent. The field is growing larger and larger every year as its popularity within computer science grows, but also as various different scientific and humanistic fields, including psychology, sociology, law, and medicine, both benefit from NLP technology and contribute to the understanding of its inner workings and role in society. ACL, one of the main NLP conferences globally, had only 258 submissions in 2002, but 3,429 submissions in 2020 , of which 279 are within the Semantics areas. ${ }^{1}$ Some of the main journals in Computational Linguistics and NLP are:

- Computational Linguistics (CL)

- Transactions of the Association for Computational Linguistics (TACL)

\footnotetext{
${ }_{1}^{1}$ https://acl2020.org/blog/general-conference-statistics/.
} 
and some of the main conferences are:

- Annual Meeting of the Association for Computational Linguistics (ACL)

- Conference on Empirical Methods in Natural Language Processing (EMNLP)

- Annual Conference of the North American Chapter of the Association for Computational Linguistics (NAACLHLT)

- Conference of the Asia-Pacific Chapter of the Association for Computational Linguistics (AACL)

- Conference of the European Chapter of the Association for Computational Linguistics (EACL)

- International Joint Conference on Natural Language Processing (IJCNLP)

- International Conference on Language Resources and Evaluation (LREC)

Also, many AI conferences have tracks or co-located smaller conferences on NLP:

- Conference on Neural Information Processing Systems (NeurIPS)

- AAAI Conference on Artificial Intelligence (AAAI)

\subsection{Competitions}

Much of the progress in NLP can be attributed to the many competitions, termed shared tasks, organized every year in various areas of NLP. In particular, the International Workshop on Semantic Evaluation (SemEval) yearly hosts several shared tasks in various areas of Semantics, including lexical semantics, meaning representation parsing and information extraction. Over the years, other important venues have also hosted shared tasks on meaning representation parsing, for example, the SIGNLL Conference on Computational Natural Language Learning (CoNLL), the International Conference on Computational Semantics (IWCS) and the International Conference on Parsing Technologies (IWPT). The datasets introduced in these shared tasks are often used as benchmarks for many years afterwards.

\section{References}

1. Bercher P, Behnke G, Kraus M, Schiller M, Manstetten D, Dambier M, Dorna M, Minker W, Glimm B, Biundo S (2021) Do it yourself but not alone: companion-technology for home improvement — bringing a planning-based interactive DIY assistant to life. Künstliche Intelligenz 35(2). https://doi.org/10.1007/ s13218-021-00721-x

2. Herbelot A, Copestake A (2021) Ideal words: a vector-based formalisation of semantic competence. Künstliche Intelligenz. https://doi.org/10.1007/s13218-021-00719-5

3. Hershcovich D, Donatelli L (2021a) Climbing the hill of computational semantics: interview with Alexander Koller. Künstliche Intelligenz. https://doi.org/10.1007/s13218-021-00718-6

4. Hershcovich D, Donatelli L (2021b) It's the meaning that counts: the state of the art in NLP and semantics. Künstliche Intelligenz 35(2). https://doi.org/10.1007/s13218-021-00726-6

5. Prange J, Schneider N (2021) Draw mir a sheep: a supersensebased analysis of German case and adposition semantics. Künstliche Intelligenz. https://doi.org/10.1007/s13218-021-00712-y

6. Pustejovsky J, Krishnaswamy N (2021) Embodied human computer interaction. Künstliche Intelligenz. https://doi.org/10.1007/ s13218-021-00727-5

7. Schiller B, Daxenberger J, Gurevych I (2021) Stance detection benchmark: How robust is your stance detection? Künstliche Intelligenz. https://doi.org/10.1007/s13218-021-00714-w

8. Shwartz V (2021) Learning high precision lexical inferences. Künstliche Intelligenz 35(2). https://doi.org/10.1007/ s13218-021-00709-7

9. Van Gysel JEL, Vigus M, Chun J, Lai K, Moeller S, Yao J, O'Gorman T, Cowell A, Croft W, Huang CR, Hajic J, Martin JH, Oepen S, Palmer M, Pustejovsky J, Vallejos R, Xue N (2021) Designing a uniform meaning representation for natural language processing. Künstliche Intelligenz. https://doi.org/10.1007/ s13218-021-00722-w

10. Yamada A (2021) The syntax, semantics, and pragmatics of Japanese addressee-honorific markers. Künstliche Intelligenz 35(2). https://doi.org/10.1007/s13218-021-00717-7 